\title{
TRATAMIENTO FISIOTERAPÉUTICO CON PROPIOCEPCIÓN Y FORTALECIMIENTO DE UN PACIENTE CON TENDINITIS ROTULIANA
}

\section{PHYSIOTHERAPEUTIC TREATMENT WITH PROPIOCEPTION AND STRENGTHENING OF A PATIENT WITH ROTULIAN TENDINITIS}

\author{
Zambrano-Chavarría Jean Carlos ${ }^{1 *}$ \\ ${ }^{1}$ Universidad Laica Eloy Alfaro de Manabí, ULEAM. Manta, Ecuador.
}

*Correo: jean-zam-3783@gmail.com

\begin{abstract}
Resumen
El trabajo propioceptivo debe ser incluido en la etapa resolutiva de una lesión y del entrenamiento, ya que es aquí donde se potencializan cada una de las cualidades necesarias para la práctica deportiva y la actividad física cotidiana. El presente estudio tiene como objetivo recuperar funcionalmente el miembro inferior afectado de un paciente con tendinitis rotuliana, mediante técnicas propioceptivas y de fortalecimiento muscular, para de esta forma reincorporarlo a las actividades de la vida diaria. La investigación fue realizada en el centro de rehabilitación de la Federación Deportiva de Manabí, Portoviejo, Ecuador. Cuando finalizó el tratamiento pudo observarse que el paciente tuvo mejoras en la fuerza, tono y resistencia muscular, así como la mejora de reflejos automáticos. La propiocepción de la articulación de la rodilla es esencial para una movilidad y estabilidad articular apropiada, puesto que procede de receptores localizados en estructuras musculares tendinosas, cutáneas y articulares.
\end{abstract}

Palabras clave: tratamiento fisioterapéutico, propiocepción, fortalecimiento muscular, tendinitis rotuliana.

\begin{abstract}
Proprioceptive work should be included in the resolution phase of an injury and training, since it is here that each of the qualities necessary for sports practice and daily physical activity are enhanced. The objective of this study is to functionally recover the affected lower limb of a patient with patellar tendinitis, using proprioceptive and muscle strengthening techniques, in order to reincorporate him into activities of daily living. The investigation was carried out at the rehabilitation center of the Manabí Sports Federation, Portoviejo, Ecuador. When the treatment ended, it was observed that the patient had improvements in muscle strength, tone and endurance, as well as improvement in automatic reflexes. Proprioception of the knee joint is essential for proper joint mobility and stability, as it comes from receptors located in tendon, skin, and joint muscle structures.
\end{abstract}

Keywords: physiotherapeutic treatment, proprioception, muscle strengthening, patellar tendinitis.

Información del manuscrito:

Fecha de recepción: 25 de octubre de 2019

Fecha de aceptación: 11 de diciembre de 2019

Fecha de publicación: 10 de enero de 2020 


\section{Introducción}

La palabra propiocepción tiene su origen en dos términos latinos propio: de uno mismo y cepción: conciencia. De esta forma la propiocepción es la conciencia de uno mismo, del propio cuerpo y del estado y posición que ocupa en el espacio. Este sistema se fundamenta en una serie de receptores de tensión y movimiento, situados en tendones, músculos, ligamentos y fascias que al ser estimulados en los movimientos envían información al sistema nervioso por medio de vías aferentes (de la periferia hacia el encéfalo) (Godes et al., 2010; Junquera, 2019).

El trabajo propioceptivo debe ser incluido en la etapa resolutiva de una lesión y del entrenamiento, ya que es aquí donde se potencializan cada una de las cualidades necesarias para la práctica deportiva y por tanto, es necesario que se apliquen desde las categorías inferiores para que las estructuras de los miembros inferiores, que son más propensas a lesionarse, adquieran fuerza $y$ proporcionen la estabilidad necesaria a las articulaciones, conllevando así, a disminuir los niveles de lesión asociados con la práctica deportiva (Aguaguiña, 2013). "En pacientes con dolor localizado a nivel tendinoso, los estudios histopatológicos detectan alteraciones en la estructura interna del tendón, que se caracterizan por un aumento de los fibroblastos, abundante colágeno desorganizado, numerosos tenocitos con diferenciación miofibroblástica e hiperplasia vascular" (Flórez et al., 2003).

En este campo de la investigación en Salud, hay que destacar los hallazgos de Croisier et al. (2001) del Departamento de Medicina Física y Rehabilitación del Hospital Universitario CHU Sart Tilman, Lieja, Bélgica. Estos autores trataron un grupo de pacientes con tendinopatías (n: 34) mediante ejercicios isocinéticos excéntricos con excelentes resultados clínicos. Realizaron un control ecográfico antes y después del tratamiento y comprobaron que las áreas con tendinosis mejoraban en un $85 \%$ y se normalizaban en un $45 \%$.

En Ecuador se calculó que en el 2012 aproximadamente el $85 \%$ de las lesiones comprometieron los miembros inferiores; el subtipo de 
lesiones más comunes fueron las lesiones de rodilla; las distensiones y desgarros de ligamentos, meniscos, cápsula articular (Aguaguiña, 2013). En lo relativo a los aspectos legales, la Constitución del Ecuador en su art. 32 , señala que la salud es un derecho que garantiza el Estado, cuya realización se vincula al ejercicio de otros derechos, entre ellos el derecho al agua, la alimentación, la educación, la cultura física, el trabajo, la seguridad social, los ambientes sanos y otros que sustentan el buen vivir.

El presente trabajo tiene un enfoque relacionado con las distintas técnicas utilizadas en la etapa de recuperación avanzada de tendinitis rotuliana. El adecuado fortalecimiento de esta zona se fundamenta en técnicas propioceptivas del miembro inferior afectado, ya que en estos casos es conveniente el fortalecimiento de los grupos musculares que rodean la rodilla, especialmente los cuádriceps, que cuentan con una función importante sobre el movimiento de todo el miembro inferior.

\section{Metodología}

\subsection{Método}

La metodología utilizada en este estudio de caso corresponde a los métodos explorativo y descriptivo, debido a que la investigación tiene por objeto describir el caso clínico propuesto a continuación.

\subsection{Caso de estudio}

El estudio realizado gira en torno a un paciente de sexo masculino de 50 años de edad, contextura endomorfa, casado, con 2 hijos, trabaja de ejecutivo en ventas en el sector privado, reside en la Ciudadela Comercio de la ciudad de Portoviejo. Además, no presenta antecedentes quirúrgicos, tiene como antecedente traumático un esguince de tobillo en julio del 2015 y no presenta antecedentes patológicos familiares.

El paciente refiere que estaba en una sesión de trote, que realiza normalmente por las noches en una pista atlética; indica que a la mitad de la sesión, mientras corría, sintió molestias al contraer el cuádriceps, posteriormente, continuó unos 500 metros y al inclinarse para colocarse en posición de sentadillas, presentó como segundo síntoma dolor, siendo 
continuo a pesar del reposo y acompañado de rigidez en la rodilla, cabe señalar que estos síntomas ocasionaron una limitación física en sus actividades diarias. El paciente decide acudir al médico Traumatólogo, solicitándole radiografía y resonancia magnética para poder deducir el problema; al cabo de unos días el paciente se reúne con el Traumatólogo para revisar los respectivos exámenes. De esta forma se pudo esclarecer la existencia de una tendinitis rotuliana. Como tratamiento para el dolor el médico envía un tratamiento farmacológico con analgésicos y antiinflamatorios, también le solicita que realice 20 sesiones de fisioterapia para la disminución del dolor y posteriormente, fortalecimiento muscular y técnicas propioceptivas cuando el dolor haya disminuido.

El paciente acude al centro de rehabilitación de la Federación Deportiva de Manabí en Portoviejo, Manabí, Ecuador, donde es evaluado por un médico Deportólogo que observa los exámenes respectivos, notando claramente la presencia de una tendinitis rotuliana en la rodilla derecha; consecuentemente, lo envía al área de rehabilitación física, en donde después de la revisión de exámenes y la valoración funcional, se concluye que se trata de una tendinitis rotuliana de rodilla derecha en un paciente de 50 años de edad.

Posteriormente, se inician las sesiones para tatar el dolor y luego tras la disminución del mismo, se realizó un tratamiento con técnicas propioceptivas, así como el fortalecimiento para el miembro inferior afectado, para de esta forma lograr un equilibrio y una recuperación exitosa.

\section{3. Ámbito de estudio}

El presente caso clínico se realizó en el centro de rehabilitación de la Federación Deportiva de Manabí, puesto que se trata de una institución que brinda sus servicios a deportistas y personas particulares, contando con los implementos necesarios para una atención de calidad. De acuerdo a las indicaciones dadas por el paciente, se conoció que el diagnóstico se relacionó con el ámbito de estudio en los puntos focales para la respectiva recuperación del caso clínico: tendón rotuliano, fuerza y volumen muscular del cuádriceps. 


\subsection{Fuentes de información}

La principal fuente de información para presentar este caso clínico es la historia clínica del paciente, que cuenta con exámenes complementarios como: resonancia magnética, radiografía, libros, sitios web y literatura científica.

\subsection{Técnicas para la recolección de la información}

Entre las técnicas empleadas para la recolección de información cabe mencionar: valoración mediante el examen físico, aplicando la inspección y palpación para determinar el origen, estado de la lesión, intensidad del dolor y establecer el estado funcional del paciente; observación de los exámenes complementarios solicitados por el Traumatólogo, tales como: resonancia magnética y radiografía. Además, se aplicó el test de valoración muscular para determinar el estado de la lesión, test del "single-leg decline squat".

\subsubsection{Implicaciones clínicas del} "single-leg decline squat"

Se trata de una herramienta versátil, de utilidad tanto en el diagnóstico como en el tratamiento, permitiendo aislar la acción del cuádriceps en el control excéntrico de la flexión de rodilla y por lo tanto focalizar la carga en el tendón rotuliano, además nos permite evidenciar una auténtica debilidad del músculo cuádriceps. La aparición de dolor entre $15^{\circ}$ a $30^{\circ}$ de flexión es indicativo de tendinitis rotuliana, mientras que a partir de los $60^{\circ}$ la carga mecánica se centra en la articulación femoropatelar y el dolor se asocia a dicha estructura. También permite regular la carga de trabajo de manera específica conjugando dos variables, el plano inclinado $\left(0^{\circ}\right.$ a $\left.25^{\circ}\right)$ y la adición de peso durante la realización de ejercicios excéntricos (Trujillo, 2012).

\subsection{Instrumentos}

Se aplicaron los siguientes test: i) prueba de Ely, para valorar la flexión pasiva de la articulación en la rodilla de forma bilateral, al notarse el aumento de la distancia entre el talón y la musculatura glútea; así como la flexión espontánea de la articulación en la cadera del mismo lado, se puede conocer el acortamiento funcional del músculo recto femoral. ii) escala de Daniels para la respectiva valoración muscular y además, se hizo uso de ligas, 
balones de fútbol, toallas, platillo de equilibrio.

\section{Resultados y discusión}

\subsection{Diagnóstico y tratamiento}

Las evidencias científicas demuestran que el entrenamiento propioceptivo muestra efectividad en tratamientos preventivos, así como en la recuperación de lesiones (Junquera, 2019).

El dolor a la palpación en el polo inferior de la rótula está siempre presente, en cuanto a las pruebas de imagen que se realizaron, el médico traumatólogo determinó mediante la resonancia magnética y la radiografía, la presencia de tendinitis rotuliana. El test de squat monopodal en declive es una herramienta útil y validada, que permite evaluar al paciente con tendinitis rotuliana; se diferencia de otros test funcionales porque tiene una mayor capacidad de aislar la acción del cuádriceps durante el squat monopodal (Flórez et al., 2003)

La aparición de dolor entre $15^{\circ}$ a $30^{\circ}$ grados de flexión es indicativo de tendinitis rotuliana mientras que a partir de los $60^{\circ}$ la carga mecánica se centra en la articulación femoropatelar y el dolor se asocia a dicha estructura (Trujillo, 2012). En el paciente evaluado en el presente estudio, el dolor se presenta a partir de los los $28^{\circ}$. Para prevenir la tendinitis rotuliana se indica al paciente que debe evitar el sobre entrenamiento, realizar estiramientos y utilizar un calzado adecuado, ya que esta fue la razón principal de la lesión.

Los síntomas presentados en el paciente reflejan que existe dolor a la palpación, engrosamiento fusiforme del tendón, o presencia de nódulos, no obstante, para establecer un diagnóstico correcto deben realizarse radiografías simples de la rodilla, para detectar la morfología y posición de la rótula. Por su parte, la ecografía y la resonancia magnética ayudan a definir el estado del tendón, permiten valorar posibles roturas o quistes intratendinosos, detectar un engrosamiento mayor 0 menor del peritendón y detectar potenciales aumentos del líquido entre el peritendón y el tendón; la resonancia además puede evidenciar otras lesiones intraarticulares asociadas.

Es a partir de este momento que se da inicio al tratamiento fisioterapéutico con propiocepción y 
fortalecimiento; en cuanto a la reeducación funcional, es un conjunto de ejercicios que tiene como finalidad reintegrar progresivamente al paciente a la vida cotidiana, a la vida social y ayudarlo a recuperar su independencia. (Dacos \& Van Bellinghen-Wathelet, 2002).

Los ejercicios propioceptivos van a dotar a la rodilla de una mayor capacidad de respuesta a los movimientos de la pierna y a la variación de cargas que ésta recibe durante la marcha, carrera, giros 0 saltos, reduciéndose así, la probabilidad de una recaída. Si tan solo se realiza un trabajo de potenciación 0 fortalecimiento muscular, pero no se integra en un patrón corporal y sistema de respuesta más funcional, la estrategia servirá de poco en la misiva de proporcionar estabilidad en la articulación (Junquera, 2010).

Habitualmente, se considera la contracción muscular como un acortamiento del músculo que ocurre cuando los filamentos de actina y miosina se deslizan entre sí, sin embargo, en algunos casos, el comportamiento muscular puede variar según el ejercicio. Estas diferencias en el comportamiento del músculo durante el ejercicio han conducido a clasificar la contracción muscular en isométrica (sin cambio de longitud), concéntrica (con acortamiento de fibras) y excéntrico (el músculo se alarga mientras se produce la fuerza). Las contracciones excéntricas son muy importantes en las actividades cotidianas y deportivas y se presentan como un elemento importante en los programas de ejercicios terapéuticos en tendinopatías (Flórez et al., 2003).

\subsection{Factores de riesgo}

En lo que respecta a factores de riesgo, se han identificado como factores intrínsecos la falta de flexibilidad de los isquiotibiales y del cuádriceps. Asimismo, se proponen los siguientes factores de riesgo: falta de dorsiflexión del tobillo, aumento en la intensidad de entrenamiento con peso, mayor masa corporal, sexo masculino, varo o valgo de la rodilla, patela alta o patela baja, ángulo $Q$ aumentado, discrepancia de miembros inferiores; aunque no existen estudios prospectivos que confirmen científicamente la relación con estos factores de riesgo (Vega, 2014). 
Por su parte, las alteraciones en la biomecánica de los miembros inferiores, así como una mala técnica de salto y aterrizaje pueden ser factores de riesgo. Está documentado que durante la primera fase del aterrizaje, un aterrizaje rígido con una disminución en la flexión del tobillo y la rodilla (rígido) puede aumentar el riesgo de tendinopatía patelar. A este tipo de pacientes se les debe enseñar a suavizar el aterrizaje. Además, se debe estudiar la densidad de las fibras nerviosas y musculares para determinar si éstas influyen en el desarrollo de esta patología.

Factores extrínsecos como canchas duras y un entrenamiento excesivo son factores de riesgo. Como ejemplo, los atletas de voleibol de playa tienen una prevalencia del $9 \%$, mientras que en los que practican en cancha dura es del 45\% (Vega, 2014).

Se logró la recuperación de la fuerza, tono y resistencia muscular, así como la mejora de reflejos automáticos en el paciente, apoyándose en los equipos de ejercicios que posee el gimnasio de la Federación Deportiva de Manabí, también se logró la mejora del equilibrio, el paciente aumentó la flexibilidad con los respectivos estiramientos que se realizaban antes y después de la sesión de tratamiento. La aplicación de estos ejercicios es una estrategia efectiva para el tratamiento de esta patología, sin embargo, cabe destacar que el apoyo de los familiares en casa fue sumamente importante para una recuperación exitosa.

Existen varios ejercicios propioceptivos que han demostrado su efectividad, desde aquellos relacionados con actividades de la vida diaria hasta métodos más sofisticados basados en plataformas informáticas; la duración de la intervención y el mantenimiento de su efecto son variables (SierraSilvestre, 2010, p.16).

La rodilla es una articulación que cumple funciones importantes, puesto que debe soportar diferentes tipos de cargas y movimientos. Muchos deportistas y entrenadores sobredimensionan su capacidad, y provocan efectos irreversibles en el deportista: aumento de las probabilidades de que ocurra una lesión; tendinitis rotuliana o llamada rodilla del saltador, debido a microtraumas por repetición. Los 
mecanismos de producción son: movilización excéntrica de la rótula, sobrecarga del entrenamiento, rótula alta y condición física deficiente (Kullak, 2009, p.5).

La propiocepción de la articulación de la rodilla es esencial para una movilidad y estabilidad articular apropiada, puesto que procede de receptores localizados en estructuras musculares tendinosas, cutáneas y articulares; la información sensitiva de estos receptores afecta a las aferentes del huso muscular. Por lo tanto, la propiocepción puede cumplir una función protectora en las lesiones de rodilla mediante protección muscular refleja (Cadena, 2012, p.9).

\section{Conclusiones}

Uno de los principales problemas relacionados con la tendinitis rotuliana es la incapacidad de realizar actividades deportivas durante un período determinado por la inflamación que sufre el tendón patelar. La tendinopatía rotuliana aparece, normalmente, por uso excesivo de la unidad musculotendinosa, causada por actividades que requieran saltos, correr intensamente, cambios bruscos y frecuentes en la velocidad, y por fuertes impactos o golpes en la rodilla. Esto se evidenció en el caso clínico analizado, puesto que el paciente que llegó por atención fisioterapéutica hasta las instalaciones del centro de rehabilitación física de la Federación Deportiva de Manabí, realizaba constantemente actividad física.

Se aplicó la propiocepción como tratamiento para la lesión de la rodilla derecha del paciente evaluado, acotando que se obtuvieron mejoras significativas en la fuerza, tono y resistencia muscular y reflejos automáticos del paciente.

\section{Bibliografía}

Aguaguiña, C. (2013). La propiocepción en el tratamiento fisioterapéutico de las lesiones de rodilla en etapa resolutiva en futbolistas profesionales del club deportivo Leon Carr del cantón Pelileo período marzojulio del 2011.Terapia Física. Universidad Técnica de Ambato. Ambato. https://repositorio.uta.edu.ec/ handle/123456789/4394

Cadena, S. (2012). Aplicación del fortalecimiento muscular $y$ propiocepción como métodos de tratamiento en lesiones ligamentosas de rodilla en futbolistas profesionales del equipo de Liga Deportiva 
Universitaria en el año 20102011. Pontífice Universidad Católica del Ecuador. Quito, Ecuador. Disponible en: http://repositorio.puce.edu.ec/ bitstream/handle/22000/5337/ T-PUCE-

5563.pdf?sequence =1\&isAllo wed $=y$

Croisier, J. L., Forthomme, B., Foidart-Dessalle, M., Godon, B., \& Crielaard, J. M. (2001). Treatment of recurrent tendinitis by isokinetic eccentric exercises. Isokinetics and exercise science, 9(2-3), 133-141. DOI: 10.3233 / IES-2001-0077.

Dacos, J., \& Van BellinghenWathelet, C. (2002). Vademecum de Kinesioterapia y Reeducación Funcional. El ateneo. ISBN 950-02-0375-8.

Flórez, M., Echavarri, C., \& Pavón, M. (2003). Programas de ejercicios en tendinopatías. Rehabilitación, 37(6), 354362.

DOI: https://doi.org/10.1016/S0048 $-7120(03) 73406-9$

Godes, J. F., Baranera, M. M., García, E. K., Parra, I. R., \& Perez, B. G. (2010). Disminución del dolor y el peso durante el tratamiento de fisioterapia en pacientes intervenidos de prótesis de rodilla. Fisioterapia, 32(1), 1116.

DOI: https://doi.org/10.1016/j.ft.200 9.08.001

Junquera, I. (2019). Ejercicios propioceptivos para fortalecer la rodilla. Propiocepción de rodilla nivel medio. Disponible en: https://www.fisioterapiaonline.com/videos/ejerciciospropioceptivos-parafortalecer-la-rodillapropiocepcion-de-rodilla-

Kullak, O. (2009). Tendinopatías en deportistas.

Asunción. pp.29,30. Disponible en: http://www.medigraphic.com/ pdfs/orthotips/ot2009/ot091c.pdf nivel-medio

Sierra-Silvestre, E. (2010). Efectividad de la reeducación propioceptiva frente a los ejercicios de fortalecimiento y estiramiento en el equilibrio, marcha, calidad de vida y caídas en ancianos. Madrid. Disponible en: http://evasierra.com/images/p ublicaciones/Articulos/Efectivi dad-reeducacinpropioceptiva.pdf

Trujillo, J. (2012). Tendinopatía rotuliana. Disponible en: https://fisioterapiayfutbol.word press.com/2012/12/07/tendin opatia-rotuliana/

Vega, A. (2014). Tendinitis patelar (rodilla del saltador). Disponible en: http://www.medigraphic.com/ pdfs/orthotips/ot2014/ot143e.pdf 